\title{
El Saber Escolar Matemático En Colombia Y La Constitución De Subjetividades ${ }^{1}$ \\ (Una Mirada Al Período Histórico 1995 - 2013)
}

\section{School Knowledge Of Mathematics In Colombia And The Constitution Of Subjectivities (A Glimpse Of The Historical Period 1995 - 2013)}

\author{
Carlos Alberto Garzón Bustos ${ }^{2}$
}

\begin{abstract}
Resumen
En este artículo se explora la relación compleja entre el saber escolar matemático y la constitución de subjetividad en el contexto colombiano durante el período 1995 - 2013. La investigación se construyó desde la educación matemática crítica (Valero y Skovsmose, 2012) como referente teórico disciplinar, a partir de herramientas metodológicas con elementos categoriales arqueológicos y genealógicos (Foucault, 1979a). Además de la búsqueda y construcción de un archivo documental, se trabajó con estudiantes de la sede de Yopal, Casanare, que cursaban el programa de Ingeniería Agronómica de la Universidad de la Salle; con ellos se recolectó información con base en la técnica denominada Grupos de Discusión. Se visibilizaron los discursos y las prácticas en los contextos sociales de encuentro entre sujetos, saberes e instituciones para rastrear el papel que juegan los saberes escolares matemáticos en la construcción de subjetividades. Se hicieron evidentes ejercicios de poder que se ejercen en las prácticas educativas con perfil matemático, generando exclusión y discriminación social. De igual manera, se describieron los escenarios biopolíticos de la educación colombiana direccionados por los intereses del mercado internacional globalizado.
\end{abstract}

Palabras clave: Matemática educativa crítica; Subjetividad; Poder; Discursos; Prácticas discursivas.

\begin{abstract}
In this research the complex relationship between schools-based mathematics and the constitution of subjectivity in the Colombian context was explored from 1995 to 2013. The research was built from critical mathematics education (Valero and Skovsmose, 2012), a theoretical discipline reference. Methodological tools and categorical elements of archaeological and genealogical type were used, (Foucault, 1979a). In addition to finding and building a documentary archive, a work study was conducted with students from the Yopal, Casanare's headquarters enrolled in Agricultural Engineering at Salle University collecting information via focus group technique. Discourses and practices in social context between individuals were visualized, the role that institutions play to dictate in construction of constitution of subjectivities. It became evident that exercises in power exerted on educational practices with mathematics profiles generates social exclusion and discrimination. Likewise, it was described the bio-political scenarios from which the Colombian education are directed by the interests of the globalized international markets.
\end{abstract}

\footnotetext{
${ }^{1}$ Este artículo se escribió con base en la Tesis Doctoral: "El Saber Escolar Matemático en Colombia y la Constitución de Subjetividades, una mirada al periodo histórico 1995" realizada por el autor en el Doctorado en Educación y Sociedad. Universidad de la Salle. 2016. Bogotá, Colombia.

2 Doctor en Educación y Sociedad. Universidad de la Salle, Bogotá, Colombia. e-mail: carlosalb985@gmail.com
} 
Keywords: Educational knowledge; Critical mathematics education; subjectivity; power; discourses and discursive practices.

\section{INTRODUCCIÓN}

"Cuéntame la historia de la génesis y desarrollo de mi entendimiento de mí mismo

[...] de tal manera que, al oirte hablar, no quiera ser más como creía que tengo que ser, y que, al oírte hablar, me dé cuenta de que esta [manera de ser], no es necesaria. M. Saar

Es importante problematizar lo que se ha venido diciendo a través de la reciente historia oficial de las matemáticas educativas en Colombia, representado en leyes y decretos; manuales y libros escolares; currículos y planes de estudio; saber escolar; encuentros de expertos, plataformas virtuales y proyectos educativos; documentos, opiniones; prácticas discursivas y no discursivas. ¿Cómo abordar el período de las últimas dos décadas en Colombia, y en él, las historias escolares matemáticas y la configuración de subjetividades de niños y niñas, de hombres y mujeres que, como en una rueda de molino, siguen atravesando y siendo atravesados (constituidos), por los discursos y las prácticas educativas? Responder la anterior pregunta tuvo que ver con el objeto de investigación abordado en la tesis doctoral que aquí se referencia; para ello se acordó una ruta metodológica.

El proceso de investigación implicó diversas fases y aspectos a tener en cuenta: por un lado, la pregunta investigativa, consistente en rastrear un proceso muy puntual. ¿De qué modo el saber matemático participa en la producción de subjetividades en el sistema escolarizador? Planteada la pregunta, se establecieron los objetivos.

\subsection{Objetivos}

General: Describir y analizar las prácticas de subjetivación que, desde el saber matemático y escolar, se ponen a funcionar en Colombia favoreciendo un tipo de gobierno sobre los sujetos.

\section{Específicos:}

1. Caracterizar los discursos del saber matemático y su relación con los modos de 
gobierno sobre los sujetos en Colombia.

2. Explorar algunos escenarios institucionalizados que sustenten las acciones educativas matemáticas.

3. Enumerar las fuerzas que han definido los fines del saber matemático escolar.

\subsection{Contextualización teórica y metodológica.}

La educación matemática crítica surge en la primera década del siglo XXI, aunque ya se habían planteado las relaciones entre la dimensión social y política con las prácticas de enseñanza de las matemáticas escolares a finales los años ochenta. Esta perspectiva de estudios busca responder a la pregunta sobre la educación matemática y su contribución a la democracia de la sociedad, mediante el confrontamiento de los discursos de dominantes, la construcción de ciudadanía y lo que sucede en las aulas y con los sujetos de enseñanza y aprendizaje de las matemáticas (Valero y Skovsmose, 2012).

Desde la perspectiva foucaultiana, la subjetividad refiere los modos en que los sujetos han sido constituidos a partir de las relaciones entre conocimiento y poder. Se accede a dichos modos mediante la objetivación del sujeto hablante. Así, la subjetividad se analiza mediante las epistemes y los dispositivos entendidos como prácticas. Tal como lo señala Castro (2011),

Las epistemes son prácticas discursivas, mientras que los dispositivos integran las prácticas discursivas y las no discursivas. El dispositivo como objeto de análisis aparece precisamente ante la necesidad de incluir las prácticas no discursivas (las relaciones de poder) entre las condiciones de posibilidad de la formación de los saberes o para analizar el modo en que se articulan las prácticas discursivas y no discursivas (p. 315).

En el proceso de investigación, la metodología se basó en el uso e implementación de la perspectiva arqueológica-genealógica, entendiendo que, "La arqueología pretende definir no los pensamientos, las representaciones, las imágenes los temas, las obsesiones que se ocultan o se manifiestan en los discursos, sino esos mismos discursos, esos discursos en tanto prácticas que obedecen a unas reglas.” (Foucault, 1979a, p. 233) Y que, “...como experiencia del orden, las condiciones históricas de posibilidades de los saberes, se propone analizar precisamente esa experiencia desnuda del orden" (Castro, 2011, p. 40.)

En este sentido, se tuvo en cuenta, en función de lo procedimental, que 
Garzón C. (2020). El Saber Escolar Matemático En Colombia Y La Constitución De Subjetividades (Una Mirada Al Período Histórico 1995 - 2013). Revista Latinoamericana de Etnomatemática, 13(4), 120139. DOI: $10.22267 /$ relatem. 20134.72

La genealogía es gris, meticulosa y pacientemente documental. (...) La genealogía no se opone a la historia como la visión altiva y profunda del filósofo se opone a la mirada de topo del sabio; se opone, por el contrario, al desplegamiento metahistórico de las significaciones ideales y de las indefinidas teleologías. Se opone a la búsqueda del origen (...) ¿Qué descubre? Que detrás de las cosas hay "otra cosa bien distinta”: no su secreto esencial y sin fecha, sino el secreto de que no tiene esencia, o de que su esencia fue construida pieza a pieza a partir de figuras extrañas a ella. (Foucault, 2004, pp. 13-18).

En consecuencia, a partir de algunas herramientas desarrolladas por Michel Foucault (Arqueología y Genealogía) se identificaron los enunciados que constituyen el archivo y que circulan por las prácticas discursivas, lo que permitió rastrear las prácticas de subjetivación que desde la enseñanza de las matemáticas han constituido y siguen constituyendo a los sujetos. De hecho, lo que se intentó fue visibilizar los ejercicios de saber-poder, amparados en los dispositivos sociales, que derivan en procesos de subjetivación en los que se hallan inmersas las comunidades educativas en Colombia. Concretamente, en nuestro caso, construimos un grupo de discusión ${ }^{3}$ (Ibáñez, 1979) con estudiantes que provienen de zonas del conflicto armado colombiano, del Proyecto Utopía ${ }^{4}$ que desarrolla la Universidad de La Salle en el programa de Ingeniería Agronómica en Yopal, Casanare.

Así las cosas, se rastreó la singularidad de los acontecimientos y los discursos escolares matemáticos en Colombia en los últimos veinte años. Se encontraron en dichas prácticas afirmaciones que refuerzan 'el orden social', la 'ejercitación sin sentido' (con mucha frecuencia), la 'higiene mental' y, donde no se reconoce el 'error' como parte de la construcción de los objetos y la comprensión de lo matemático; lo que parece, en conjunto reforzar la exclusión en beneficio de la explotación; todo lo anterior como si la 'verdad

\footnotetext{
${ }^{3}$ El grupo de discusión es un grupo simulado y manipulable. Simulado porque solo es la esperanza de que sea un grupo, solo se reúnen mientras hablan, su espacio de encuentro es efímero pues dura en tanto la conversación se desarrolle. Manipulable porque es el preceptor quien tiene el poder de asignarle la palabra y el tiempo a los sujetos convocados. Es él quien determina el grupo y decide cuando nace y muere (Ibáñez, 2011, p. 271-272)

${ }^{4}$ Este proyecto líder en la Universidad de la Salle tiene como propósito convertir jóvenes bachilleres de zonas rurales afectadas por la violencia en Ingenieros Agrónomos con la mejor formación posible y con la metodología 'aprender haciendo' y 'enseñar demostrando', hacerlos líderes para la transformación social y política, y la empresarización productiva del campo en sus lugares de origen como resultado del proceso. Todos los estudiantes están becados durante los cuatro años de su carrera. A lo largo de ella y como trabajo final los estudiantes desarrollan una propuesta de Proyecto productivo que deben implementar en su lugar de origen. Tomado de: https:/publica.lasalle.edu.co/wcm/connect/e2865643-c83b-4402-8380$\underline{17659402574 \mathrm{c} / \text { Programa_Apasionados_por_la Tierra.pdf?MOD=AJPERES\&CVID=mWppi5a }}$
} 
matemática' fuese única y ya estuviese garantizada en el uso y aplicación del texto escolar y desde la práctica del profesor, y avalada por las instituciones educativas como verdad indiscutible que jerarquiza los posibles lugares sociales del sujeto y sus relaciones con el mundo que habita.

¿Efectivamente las matemáticas escolares constituyen sujetos críticos y autónomos? ¿Son, entre otras cosas, las matemáticas escolares en Colombia, también, un instrumento biopolítico? ¿Hacen parte estas prácticas escolares de la construcción social de un tipo de racionalidad? Lo que exigió reflexionar acerca de lo que se comprende por quehacer matemático, desde donde volvimos a preguntar: ¿Caben en las prácticas reales de los escenarios escolares las experiencias cotidianas de los estudiantes?

Y si, como afirma Foucault (1979b), el saber no está hecho para comprender, sino para zanjar, así, la pregunta es por el tipo de saber matemático escolar que circula en Colombia, por ese saber que a partir de ciertas fuerzas en juego contribuye a constituir en un momento particular un tipo de sujeto-s (subjetividades). Así, ¿De qué modo ese saber matemático se relaciona, por ejemplo, con las evaluaciones internacionales de matemáticas? ¿Cuál es esa historia que no ha emergido en las investigaciones en educación matemática en Colombia, y que hasta hace muy poco empieza a dibujarse?

No es necesario volver a decir que las matemáticas han jugado un papel importante en la historia moderna, es de común aceptación su inclusión en los currículos escolares en todo el mundo; sin embargo, interesó principalmente ahondar en los discursos y las prácticas escolares y su procedencia, "la cual permite también reconocer bajo el aspecto único de un carácter, o de un concepto, la proliferación de los acontecimientos a través de los cuales (gracias a los cuales, contra los cuales) se han formado" (Foucault, 2004, p. 26) los sujetos. Situaciones que se han naturalizado, como, por ejemplo: formas de comportamiento al hacer clases de matemáticas; formas de evaluación de los saberes; clasificación de los sujetos a través de pruebas estandarizadas; discursos supuestamente lógicos y científicos para soportar decisiones; didácticas y pedagogías ancladas en la continuidad y la repetición. Cabe, entonces, preguntarnos por las consecuencias de este tipo de prácticas escolares en Colombia; ¿Quiénes son promovidos en la evaluación de los saberes escolares matemáticos? ¿Cómo y por qué se utilizan unos textos, unos lenguajes, unas didácticas, en 
Garzón C. (2020). El Saber Escolar Matemático En Colombia Y La Constitución De Subjetividades (Una Mirada Al Período Histórico 1995 - 2013). Revista Latinoamericana de Etnomatemática, 13(4), 120 139. DOI: $10.22267 /$ relatem. 20134.72

los escenarios escolares? ¿A quiénes les es lícito hablar de las matemáticas escolares?

En la primera parte de este artículo hay una intersección compleja entre un régimen de verdad amparado en una visión estrecha del saber matemático, y un conjunto de prácticas en medio del contexto violento que nos ha acompañado en los últimos cincuenta años. Igualmente, se muestra parte de otra historia no oficial de los sujetos escolares: en ejercicio educativo; configurándose como sujetos estudiantes, en los escenarios de enseñanza y/o aprendizaje. Un conjunto de prácticas biopolíticas en la arena movediza sociopolítica y económica del país.

Para continuar descubriendo el régimen de verdad del saber matemático escolar, en la segunda parte, se muestra la empobrecida relación entre el docente y el saber matemático. Aquí las fuentes primarias mostraron los enunciados referidos a las matemáticas como parte fundamental del discurso escolar: las matemáticas se deben enseñar porque 'forman el pensamiento' y por su utilidad en cualquier campo.

Luego, en la tercera parte, aparece una descripción de las instituciones que, desde el marco estatal y normativo, gobiernan las prácticas y discursos educativos matemáticos, lugares donde discurre la vida de los sujetos docentes y estudiantes, en el contexto de la sociedad colombiana y dentro del escenario neoliberal del mercado globalizado, a lo que se ha denominado la vorágine de las pruebas donde se clasifica a los estudiantes como brutos y/o perezosos. Se avanza en la reflexión del contexto de mercado globalizado donde ocurre la educación matemática en Colombia.

La cuarta parte muestra algunas relaciones entre el saber matemático y la educación en Colombia, los escenarios educativos y las metas a conquistar en la enseñanza oficial, para finalizar con una reflexión sobre la enseñanza como práctica discursiva y ejercicio de poder.

\section{RESULTADOS}

\subsection{Sujetos y educación}

Las prácticas y discursos de la educación matemática permiten fabricar al sujeto racional, objetivo, universal que realiza 
Se advierte que en torno a las matemáticas existen discursos que se han naturalizado a través de las prácticas, desde donde se supone que lo que está asociado con las matemáticas es difícil, por no decir imposible, y además que es una disciplina propia del mundo de los muy inteligentes, lo que ha generado (y, sigue generando) exclusión y marginación. En efecto, es indudable la manera como se ha mitificado el aprendizaje de la matemática, haciendo creer que es un campo de estudio exclusivo para los superdotados de inteligencia, tal como se evidencia en la voz de un estudiante de Yopal,

Cuando había evaluaciones de matemáticas, casi siempre las perdía; se alegraban (los profesores) [...] si como que ellos ya estaban, ya habían establecido qué estudiantes más o menos pasaban, eran inteligentes y, otros que no lo éramos tanto; lo normal era que perdiéramos.

De otro lado, se afirma que la dificultad está del lado de los estudiantes y su capacidad reducida o recortada que no es susceptible de mejora.

De acuerdo con lo anterior, se puede advertir la construcción de un régimen de verdad que se ha convertido en el referente natural de los discursos educativos sobre la enseñanza de lo matemático en Colombia; se ha acostumbrado a creer y actuar como si el aprendizaje de las matemáticas fuera un campo de estudio solo para los muy inteligentes, y la aparición del miedo como una categoría existencial relevante en las experiencias matemáticas. Cabe decir que a partir de la docencia (y, no solo de la docencia; veremos [tercer movimiento] otras fuerzas discursivas), matemática en Colombia se ha generado una situación enmarcada en la

frustración y la falta de participación interesada en aprender matemáticas [...] la enseñanza no atrapa a los estudiantes, sino sobre todo que está lejos de poder contribuir a la equidad social, al establecimiento de conexiones con la vida diaria y a la democratización de las relaciones sociales en nuestro país (Valero, 2006, p. 2).

\subsection{El docente y el saber matemático: Una relación empobrecida}


En cuanto a los profesores de matemáticas y su ejercicio académico, conviene detenernos un poco para recordar algunas palabras de Zalamea (2014) a propósito de las matemáticas en Colombia, lugar donde se empieza a reconocer que el aporte de los matemáticos colombianos, comparado con el de los matemáticos de otras latitudes, en los últimos 400 años es casi nulo; en el caso del aporte de los profesores de matemáticas colombianos, en comparación con la investigación en educación matemática, ocurre lo mismo. Entonces: ¿De qué, o de cuál saber matemático es que se habla, dice, enseña? Posiblemente del saber matemático que se ha institucionalizado a través de los discursos y las prácticas escolares. Pareciera que se nos ha condenado a repetir hasta el cansancio sin reflexionar sobre lo que se hace, y nada más. Repetir sí tiene que ver con la disciplina matemática, por cierto; pero lamentablemente nos quedamos solo en eso; en una repetición insulsa. Esta frase de un estudiante, lo corrobora,

En octavo tuve un profesor que se enredaba él mismo y no sabía explicar las ecuaciones y yo nunca pude entender qué era una ecuación, porque él las colocaba en un tablero y se ponía bravo cuando le pedíamos explicación, entonces dejaba un trabajo y en la otra clase teníamos que traerlo resuelto; nosotros simplemente buscábamos el álgebra y copiábamos y listo. [...] Y acá me ha tocado sufrir.

Cabe decir, además, que lo que se llamó el desarrollo en los países del tercer mundo a partir de la segunda mitad del siglo XX, - y, que se consignó en las apuestas de los organismos internacionales, como el Banco Mundial, el FMI, (Escobar, 2007, pp. 55-56)- igualmente se advierte en los ejercicios educativos con perfil matemático, y de qué manera. Se detecta que se continúa con la repetición poco crítica de las tendencias impuestas desde los centros de poder -capitalismo cognitivo- orquestadas por las editoriales (y, ahora los vendedores de software), promovidas en los encuentros académicos con los consabidos expertos, amparados por los lineamientos y decretos institucionales, en acciones que parecen renovar las reflexiones sobre la enseñanza disciplinar, pero que a fin de cuentas resulta ser un ejercicio de confirmación de un estado de la misma. Esto es, la jerarquización y la inequidad como rasgos destacables para aprendizajes escasos, cuando no nulos.

\subsection{La educación matemática y la vorágine de las pruebas}


Ahora bien, con base en lo que se ha venido mostrando, se advierte que los estudiantes no consiguen superar las evaluaciones institucionales y las pruebas estandarizadas (PISA, Saber Pro) con un nivel aceptable de competencia matemática por nivel, entonces, ¿Qué es lo que ocurre en esta triada: profesor, estudiante, ¿Saber matemático? ¿Qué es lo que se ha naturalizado en esta relación? Quizás, el no haber revisado esta relación y su contexto socio político (Valero, 2002) es, en parte, lo que sigue generando procesos de exclusión y marginación desde la educación matemática.

¿Cómo abordar esta situación? ¿Cómo superar el esquema (régimen de verdad) que nos muestra el desarraigo y los 'pobres' resultados académicos de tantos estudiantes? Habría que empezar a construir una historia menos oficial, efectiva (Foucault, 2004, p. 210), del saber matemático en Colombia. Unido a lo anterior (Valero y Skovsmose, 2012), encontramos que la educación matemática en Colombia no aporta a la construcción de la democracia. La enseñanza de las matemáticas se soporta en el poder de su certeza, lo que no permite la menor sospecha de los ciudadanos (Vithal y Valero 2012).

\subsubsection{Estudiantes brutos y/o perezosos}

Esta clasificación despiadada se ha naturalizado, se ha convertido en parte de una narrativa cotidiana que sigue alimentando los mitos acerca de las matemáticas en nuestro país. Situación que ha invisibilizado otras sensibles variables que afectan los procesos educativos en Colombia, tales como el contexto social y cultural, la discriminación y la exclusión, el género y la diversidad de los sujetos. Cabe decir que los y las estudiantes siguen haciendo parte de una clasificación por edades (y, supuestos) desarrollos cognitivos enmarcados en planes de estudio desde los escenarios de la educación infantil, pasando por la educación básica y media hasta llegar a la llamada educación superior. En todos estos niveles lo que aparece como relevante es que los estudiantes poco aprenden.

Encontramos que las pruebas determinan el ingreso a la educación universitaria; sin unas competencias mínimas en lectura y escritura, como sin el manejo básico de las herramientas matemáticas, es casi imposible construir otros escenarios de formación para participar en una sociedad moderna y global, situación que afecta ostensiblemente los resultados escolares. Como se ha señalado, la existencia del estudiante en la institución y 
Garzón C. (2020). El Saber Escolar Matemático En Colombia Y La Constitución De Subjetividades (Una Mirada Al Período Histórico 1995 - 2013). Revista Latinoamericana de Etnomatemática, 13(4), 120139. DOI: $10.22267 /$ relatem. 20134.72

en el aula, y las variables que inciden en su aprendizaje están reguladas por relaciones complejas entre el docente, el contexto y él mismo, y mediadas por lo cultural, lo político y lo social, lo que pone en entredicho los ideales escolares y la idea de carácter lineal entre asistencia, intereses, ejercitación y aprendizajes indudables.

Se podría inferir, que las causas (entre otras posibles) de los limitados resultados académicos de los y las estudiantes en Colombia de los estratos socioeconómicos más vulnerados, tienen que ver con la inasistencia estatal y el contexto escolar, en efecto, situaciones como la descrita por una estudiante del Programa de Ingeniería Agronómica, en Yopal: "mi colegio era totalmente abandonado por los entes gubernamentales del departamento, con decirle que no tenemos salones dignos, nosotros estudiamos en chozas hechas por nosotros mismos y los padres de familia”. Esta situación se repite en muchos escenarios educativos en Colombia.

\subsubsection{Matemáticas y competencias estudiantiles: Un asunto de mercado}

A propósito de pruebas internacionales y competencias, nos podemos preguntar ¿Qué es esto de las competencias matemáticas? Además de una declaración de 'buenas intenciones' de los gobiernos y las agencias económicas, habrá que examinar los enunciados que se han naturalizado por el uso y las prácticas discursivas de nuestro entorno educativo. Si de lo que se trata es de alfabetizar matemáticamente a los sujetos (estudiantes en este caso), hay que recordar que Michael Apple "ha distinguido dos tipos de alfabetismo: funcional y crítico" (Valero y Skovsmose, 2012, p. 65). En términos de lo funcional, las competencias deberían servir para que las personas cumplan con tareas mecánicas y repetitivas, lo que interesa al mercado para progresar en sus intereses económicos; pero, desde el punto de vista de la alfabetización matemática crítica, las competencias deberían servir para que los sujetos participen en las decisiones políticas y puedan, a través de las herramientas matemáticas, ayudar a construir otros mundos; mundos ojalá más cercanos a las complejas realidades de los países de América Latina.

Declaraciones como: "crear un clima de interacción y reconocimiento multicultural en el aula, propicio para la actividad del estudiante desde su saber ser [...] favorable al desarrollo de competencias matemáticas" (García, Coronado y Montealegre, 2011, p. 167), 
necesitan ser consideradas con más atención y menos inocencia cómplice; con todo y lo anterior habrá que cuestionar (Gentili, 2015, p. 23) las pruebas PISA y las competencias al estilo del mercado funcional.

Cabe anotar que los contextos inciden notablemente en la vida de los estudiantes, y esto se manifiesta en las instituciones escolares y su dinámica compleja. En el caso colombiano los conflictos de la guerra y la pobreza urbana y rural no han sido considerados seriamente en la educación matemática; de hecho, la estadística como herramienta matemática, como "poder - saber fundamental en la construcción histórica del mundo"17 hace parte de la producción y mantenimiento de conflictos sociales y políticos, lo que tiene una raíz fuerte (mejor, un rizoma, al decir de Gilles Deleuze) en la constitución de la ciencia occidental que está soportada en buena medida en la modelación y la lógica matemática.

Visibilizar los conflictos sociales en las historias escolares se hizo necesario; las desigualdades, las injusticias y la violencia también hacen parte de las aulas y la educación matemática, y en muchas de ellas los estudiantes mismos los personifican. Los escenarios habrán de ser abordados con otras lógicas, otros lenguajes, otras sensibilidades. Tema urgente para una posterior investigación en educación matemática y posconflicto en Colombia.

Un caso relevante, se encuentra en varios testimonios de estudiantes de la Sede del Yopal, de la Universidad de la Salle:

En el colegio yo era personero estudiantil y un muchacho que estudiaba conmigo en el mismo grado se fue para la guerrilla y los convencen y otros se van porque no tienen trabajo, otros por la familia. Si el papá fue guerrillero entonces los hijos también [...] Donde yo vivo están los Elenos y los de las Farc, principalmente. Por ideologías, por el territorio, por cualquier cosa tienen conflictos y el que paga es el pobre campesino. Si usted no le colabora al uno le tiene que colaborar al otro, y si el otro se entera es un problema, entonces usted no sabe si coger para allí o para acá

\subsection{El saber matemático y la educación}

En un enlace fascinante entre la plasticidad y la precisión, el matemático debe ayudar a fraguar y criticar una época tan desorientada como la nuestra, donde se exacerba un uso acrítico y poco riguroso de las variedades del saber.

Fernando Zalamea 
En el libro Fundamentos de Matemáticas, encontramos algunos elementos necesarios y suficientes para retomar el 'significado de las matemáticas'. No se trata, por supuesto, de una búsqueda exhaustiva acerca de lo que significan hoy las matemáticas en el mundo de la segunda década del siglo XXI; la libertad y la imaginación (Zalamea, 2007, p. 123) construyendo hipótesis, soportadas y contrastadas a través de definiciones, teoremas y ejemplos es lo que significan, en alguna medida, las matemáticas. Por ello, es conveniente situarnos en el contexto de las matemáticas escolares en Colombia donde se evidencia que -en la gran mayoría de los casos- lo que se hace es repetir y eso tan solo una parte muy delimitada y muy pequeña de ese mundo vivo y en crecimiento permanente llamado Matemáticas.

En otras palabras, la enseñanza de las matemáticas pone al aprendiz en dinámicas en las que prima la contradicción entre lo que se dice y lo que se hace, olvidar lo que se es para acceder a lo que se debe ser, a lo que le exigen las lógicas del mundo del mercado en el que debe participar y en el que ha de representar, al menos, el papel de sobreviviente de los procesos educativos. En la práctica escolar se evidencia que todo, o casi todo se puede solventar teóricamente en términos de derechos, de cobertura, de inclusión para la educación. Lo que abre interrogantes sobre lo que se pone en juego tras la enseñanza de las matemáticas para, por un lado, sustentar discursivamente las condiciones y objetivos de los procesos en términos democráticos, y por el otro, para restringir el acceso al conocimiento desde las mismas prácticas, generando diversos tipos de subjetividades producidas para la poca reflexión y la aceptación de lo que impulsa el mercado, subjetividades que facturan la clasificación orientada a la segregación con fines sociopolíticos más conocidos que ocultos.

\subsubsection{Las matemáticas y el desarrollo del pensamiento.}

La insistencia en la racionalidad (un tipo de racionalidad), "el supuesto de la resonancia intrínseca de las matemáticas y la educación matemática [...] dentro de la imagen del estudiante como sujeto cognitivo," (Valero, 2002, p. 55) aparece como la meta a conquistar a través de la enseñanza de las matemáticas. Pero "los educadores, al enseñar matemática, están enseñando no sólo un contenido, sino un modo de aprender contenidos; 
esto es, una metodología de interactuar en el mundo.” (De Moura, 2011, p.54). La escuela destituye, de manera arbitraria y en nombre del 'pensamiento científico', lo que es diferente a él mismo,

Al iniciar a los estudiantes en la actividad científica, se les pide renunciar a su modo de conocimiento habitual; [...] el acto pedagógico violenta a los estudiantes cuando impone conocimientos que aparecen bien sea como resultante de un arbitrario cultural [...], o que no interesen a los estudiantes, o que los fastidien, ya que se oponen a las representaciones que se habían construido. (...) se trata de una demanda de conversión y se percibe que se trata de imponer no sólo conocimientos, sino también un modo de conocimiento que se construye contra el sentido común, (...) se pretende pedirle al estudiante que transforme su subjetividad en objetividad, lo que equivale a valorizar el objeto a costa del sujeto, cuando justamente la búsqueda identitaria del estudiante le lleva a afirmarse mediante la expresión de sus propios valores, sus propias opiniones, su cuerpo y sus sentidos... otras tantas dimensiones que la actividad científica pide abstraer (Trabal, 2011, p. 231)

Constituyendo, así, prácticas que sustituyen la subjetividad que le es propia a cada estudiante por la supuesta 'objetividad científica' en un trueque que proscribe el pensamiento y sus transformaciones; lo objetivo sumado a la objetividad no puede derivar en algo diferente a la 'certeza', con lo que se consolida el carácter infalible y definitivo de la enseñanza de las matemáticas.

No es un secreto que, cada vez más, la escuela pierde su lugar como espacio de saberes; esto no es reciente, tan solo ocurre que la atención de analistas y teóricos se agolpó en la institución escolar hasta hacer visible este hecho social y cultural, lo que no quiere decir que la escuela no siga cumpliendo con sus funciones en esas órbitas de interacción entre sujetos. La escuela no sólo descontextualiza los saberes, sino que no dota de sentido su hacer, seguramente porque ese sentido, cuajado de intencionalidades invisibilizadas de orden político, no atañe a los sujetos que allí concurren, hay que decirlo, decisión de las sociedades.

Hace un buen tiempo, quizás, 'había claridad' sobre lo que implicaba ser inteligente, tener capacidad de inteligir. Hoy, es claro, esta facultad no está (únicamente) determinada por logros en matemáticas, por ejemplo, aunque la clasificación social de los sujetos sigue estando atada a esa mitificación. ¿Por qué se mitifican las matemáticas y su enseñanza? Un detalle de esto es el lugar y reconocimiento que tiene el profesor de matemáticas frente a 
docentes de otras asignaturas, tanto en el colegio como en la universidad.

Se puede decir, a partir de lo anterior, que la experiencia con las matemáticas afecta la vida de los sujetos como estudiantes, principalmente, y por supuesto, su vida posterior. Los vínculos con lo matemático dependen de experiencias que lo sean, es decir, de que lo abordado en la escuela y fuera de ella represente transformaciones en la manera de percibirse y percibir el mundo. Y las experiencias, en este sentido, escasean en al ámbito escolar. Las deficiencias tomadas como oportunidad para reforzar aprendizajes, más que para sancionar modificaría, quizás, las condiciones de aprendizaje, pues a las dificultades de la vida cotidiana no se sumarían la certeza, esta sí fundamentada, del fracaso escolar.

\subsubsection{Prácticas matemáticas y ejercicios de poder}

Las prácticas pedagógicas, tanto como las de aprendizaje ponen al sujeto en un lugar que es visible para la sociedad, tanto si se trata de logros, como de deficiencias, especialmente en lo que toca a los estudiantes. Y se enseña o se aprende desde los supuestos que sobre las matemáticas circulan, por ejemplo, su rasgo de racionalidad incuestionable. Un mínimo de conciencia sobre el hacer del docente y sus implicaciones podría generar un giro en las realidades poco halagüeñas de los aprendices, conciencia en paralelo a un acercamiento a la importancia social y de exclusión posible que vehiculan sus prácticas.

Con todo, es difícil cambiar posturas y concepciones en tanto se trata de encuentros cotidianos de subjetividades y su complejidad. Por otra parte, y de manera efectiva, en su circulación social y cultural las matemáticas no son solamente una disciplina, pues, "el hecho de que a través de las matemáticas se transmitan valores como la racionalidad, la objetividad y la precisión, entre otros, las hacen un conocimiento privilegiado para poder actuar dentro de un mundo que valora estas cualidades." (Valero, 2006, p. 10). En efecto, se constituyen en escenario en el que se ofertan valores que, como un sortilegio para iniciados, abre y cierra puertas negando y concediendo favores según la apropiación efectiva de estos valores. De ahí la importancia de la enseñanza y de la conciencia sobre qué se enseña, cómo y para qué.

Tener las capacidades de discernir, argumentar, abstraer y sacar conclusiones faculta 
para la toma de decisiones. Y la democracia es, por definición, una manera de ser de lo social, basada en la participación colectiva. Con base en los criterios que podría proveer la formación se estaría hablando de una sociedad con recursos participativos que incidirían de manera sensible en sus rumbos.

Los lineamientos y las políticas no escasean en la justificación de lo que es necesario tener en cuenta para una enseñanza adecuada que evalúe a partir de cánones establecidos, los logros y niveles de aprendizaje alcanzado, aunque no se logre establecer, o no se pueda saber qué es lo adecuado en lo que corresponde a la educación.

De esta manera, el MEN parte de lógicas en las que están presentes la secuencialidad, el desarrollo y lo sucesivo, todo concebido como incuestionable manera de mejorar, tanto en la enseñanza como en los aprendizajes. Cabe señalar que no se comprende por qué la enseñanza se escribe en singular y los aprendizajes en plural. Quizás se trate del eco de las intenciones de unificación del quehacer docente que se proponen desde la escuela, justamente como objetivación racional de la enseñanza, aspecto que cuestionamos aquí.

No faltan, sin embargo, especificaciones para que se trabaje de manera concienzuda y gradual desde los estándares para matizar las metas con los tiempos destinados a su alcance. Frente a la rigidez de los estándares se propone una fluctuación de tiempos y una puerta para el ritmo singular de los aprendizajes. Metas concretas e inamovibles, sí, pero en la flexibilidad del hacer en el tiempo y con tiempo.

Esto habla claramente de la concepción de ciclos de aprendizaje, pensados, entre otras cosas, para disminuir la deserción y el fracaso escolar. Y, claro, de una separación necesaria entre el tiempo de apropiación de contenidos y el tiempo escolar. Pero esto es algo que se queda en las posibilidades de las determinaciones ministeriales -que parecen sin límite a la hora de formular prescripciones-, por efecto del desconocimiento sobre las dinámicas escolares y los contextos que las sustentan. En la enseñanza, y no solamente de las matemáticas, la presión del tiempo y los resultados, los 'mejores', siguen echando por tierra las buenas intenciones del estamento institucional. Aunque no sean sólidos los saberes adquiridos, o sean escasos, superficiales, trivializados, la meta parece ser el logro de cohortes de estudiantes graduados que justifiquen los esfuerzos institucionales por la 
cobertura, aún en desmedro de la calidad. Aunque no logren aprender matemáticas, ni otras ramas del saber. Lo mejor y lo más rápido, términos con frecuencia excluyentes, dominan el panorama de los afanes educativos.

Por otra parte, la pregunta es cómo lograr transformaciones en la enseñanza de las matemáticas cuando está regulada por prácticas que la atan al 'siempre lo mismo' en tanto se trata de 'verdad' y 'certeza' de las que se invisibiliza el cuestionamiento sobre su condición y estatus social. Resulta, cuestionable, con todo, el asunto de los 'consensos básicos' para adelantar procesos de transformación de la educación, no solamente en la educación superior. Se advierte, no obstante, que éstos no pueden (únicamente) derivar de lineamientos y políticas, sino que requieren, para un mínimo de aceptación y circulación social, de su emergencia en los procesos mismos de formación. Su función más visible es la de recordar al profesor que existe un contrato entre su saber y sus estrategias, el tiempo del que dispone y sus grupos de estudiantes.

Se evidencia que, a partir de las realidades que crean la necesidad de la reflexión y el diseño de estrategias que sustenten la toma de decisiones en torno a lo educativo, se propone fortalecer la oferta educativa para las poblaciones vulnerables, pero visto el estado actual de la educación, especialmente en matemáticas, la idea de las poblaciones vulnerables aplica para la gran mayoría, lo que vuelve norma la expresión, no excepción. Y de hecho tendría que ser norma: basta revisar lo que corresponde a la enseñanza en lo rural, los enormes problemas asociados a la pobreza y la violencia revelados, por ejemplo, en las entrevistas realizadas en Yopal, evidencias que se recopilaron en el trabajo realizado con estudiantes de la Universidad de la Salle mostrando lo que ocurre en la educación matemática rural.

En su condición subjetivante de 'verdad' incuestionable, las matemáticas como instrumento de parcelación del cuerpo social no parecen un campo dispuesto a hacer concesiones, ni siquiera en la pedagogización de la que es objeto en la escuela. Y, de manera paradójica, quizás es en ella donde mayor es su fuerza de 'verdad', mientras que, como saber, abre espacios a la pregunta y a la investigación gracias a la incertidumbre que generan las fisuras de su arquitectura. En la enseñanza es dogma. $Y$ un dogma que requiere de pocos elementos de doctrina para establecerse en la cumbre de los saberes escolares, lo que no es gratuito ni carente de ideología. Y, la enseñanza de las matemáticas con niveles de 
aprendizajes escasos o nulos, tiene que ver con este descenso, sin duda dramático, del rendimiento, término asociado también a la producción neoliberal. Enunciado en perspectiva de necesidad y deber, el discurso se torna meramente prescriptivo, a la vez que revela lo que hay de trasfondo al ligar aprendizajes y rutas, las que sean, de la vida de los estudiantes.

Lo que ocurre en las aulas de alguna manera favorece el escape de esas planificaciones institucionales; el ministerio se queja porque los resultados educativos no responden aún a lo proyectado, aunque el supuesto cumplimiento y la efectiva desviación, en su ocurrencia, tienen un alto costo. En este sentido, la educación matemática crítica podría presentar una alternativa a este callejón educativo.

\section{CONCLUSIONES}

Porque no hay palabras, sino una larga humillación del no decir. Carlos Skliar

Una vez recorrido este trayecto rizomático, en medio de los meandros de una historización (no oficial) de un periodo (1995 - 2013) de la educación matemática en Colombia, nos quedan muchas preguntas, quizás, algunas respuestas; $\mathrm{y}$, una enorme veta de investigación por desarrollar. En efecto, con este trabajo se intentó visibilizar esos regímenes de verdad (Foucault, 1979a) que envuelven las prácticas escolares matemáticas, $\mathrm{y}$, que han constituido, en parte, la subjetividad de los seres de carne y hueso llamados estudiantes y profesores.

Regímenes de verdad soportados, sofísticamente, en el supuesto y 'verdadero razonamiento', como si esto pudiese existir, cuando hace más de 100 años las matemáticas explotaron en un mundo de infinitas posibilidades, desembocando en el portentoso teorema de Gödel; pero, se sigue enunciando un tal razonamiento matemático único, y lo que es más irresponsable, sancionado a muchos niños y niñas, jóvenes y adultos en situación escolar. De igual manera, con este único y supuesto razonamiento matemático, se intenta (iy se consigue!) justificar la modelación del mundo financiero soportado en la economía, se soporta la inversión tecnológica y la investigación puesta desde los intereses globalizantes del mercado que, como ejercicios de poder, producen exclusión y marginación. 
Paralelamente, se van configurando subjetividades y sujetos atemorizados. De ahí la urgencia en seguir reflexionando desde una matemática educativa crítica.

Hemos hecho un viaje a través de la construcción de un archivo (Foucault, 1979a); archivo constituido y constituyente en el escenario histórico social de las prácticas escolares matemáticas en el periodo comprendido en este trabajo, lo que nos ha mostrado un conjunto de discursos, efectivamente puestos en las dinámicas escolares; instituciones y políticas; sujetos que aprenden; sujetos que enseñan; y, un saber escolar matemático, han sido los referentes categoriales que nos permitieron vislumbrar la constitución de subjetividades que fecundan el miedo y en muchos casos la desesperanza tal como lo señalan los estudiantes Yopal. Para seguir reconociendo la oscuridad impuesta en América Latina del legado moderno y colonial en la educación, puesta ahora, en la dirección del mercado neoliberal.

Se han escuchado las voces de los excluidos y discriminados en nombre de la razón positiva del saber matemático oficial, en medio de sus contextos y experiencias, enfrentando con dignidad la paquidermia estatal - institucional con sus rituales inflados de burocracia y recomendaciones superficiales. Es hora de hacer un alto en el camino, y procurar un rescate de lo humano, para empezar a superar "las tendencias mecánicas clasificatorias que aprisionan al pensamiento en el lenguaje de la lógica proposicional.” Zemelman, H., (2012: 44).

Si bien, hace más de un siglo, las matemáticas superaron la frontera lógica proposicional bivalente (Zalamea, 2010), igual que la linealidad positiva de las ecuaciones diferenciales clásicas, la situación no ha llegado a la educación matemática. Se siguen enseñando y promoviendo unas 'matemáticas chiquitas' que posiblemente, no son las más 'ajustadas' a los escenarios complejos de las realidades colombianas. Se espera que una causa tenga el mismo efecto, como en el sueño laplaciano moderno de la física clásica, y se ignora que existen mundos paralelos (mecánica cuántica) y otras maneras de ser - siendo.

Se hizo un análisis híbrido de las macro categorías: Sujetos, Saber Escolar Matemático e Instituciones y con el fondo de algunas narrativas (Grupos de Discusión, $\mathrm{N}^{\circ}$ 1,7); de no pocos sujetos excluidos y discriminados en nombre de la razón positiva del saber matemático oficial, en medio de sus contextos y experiencias. Emergió allí la paquidermia 
estatal - institucional, disponiendo de la vida de los sujetos; ejercicio de gubernamentalidad (Castro, 2010) escolar matemática que excluye, margina e invisibiliza desde las regiones y los contextos sociales colombianos (sobre todo, los de mayor pobreza y violencia) las complejas realidades que ocurren en los escenarios educativos.

Se sigue asumiendo impunemente que unos estudiantes (pocos) 'saben', generalmente los que pertenecen a los estratos altos, porque superan una ( $\mathrm{o}$, varias) prueba estandarizada, y, otros (muchos) 'no saben'. Lo que ahonda la brecha social entre los que pueden apostar por un futuro de esperanza y posibilidades de desarrollo humano, y los que son condenados desde muy jóvenes a la pobreza y la exclusión social. Una pregunta de acuerdo con lo anterior, podría ser: ¿Qué es lo que saben estos estudiantes? o ¿Qué es lo que no saben los otros? Habría que ir más allá, para preguntarnos, por el sentido de tantos discursos y prácticas escolares, desconfiando de la naturalización del lenguaje en que vivimos, o en el que creemos a fuerza de la subjetivación escolar, que es promovida, ahora, hipertextualmente por el marketing globalizante y sus intereses insulsos.

\section{BIBLIOGRAFÍA}

Castro, E. (2011). Diccionario Foucault, Temas, conceptos y autores. Buenos Aires: Siglo XXI.

Castro, S. (2010). Historia de la gubernamentalidad. Bogotá: Siglo del Hombre Editores. Universidad Javeriana.

De Moura, M. (2011, enero-abril). Educar con las matemáticas: saber específico y saber pedagógico. En: Revista Educación y pedagogía, Universidad de Antioquia, 23 (59), 47-57.

Escobar, A. (2007). La invención del tercer mundo. Construcción y deconstrucción del desarrollo. Venezuela: Fundación editorial el perro y la rana. Recuperado de https://bibliotecapromocion.msp.gob.ec/greenstone/collect/promocin/index/assoc/H ASH018c.dir/doc.pdf

Foucault, M. (1979a). La arqueología del saber. Barcelona: Siglo XXI.

Foucault, M. (1979b). Microfísica del poder. Madrid: Ediciones la Piqueta.

Foucault, M. (2004). Nietzsche, la Genealogía, la Historia. Valencia: Pre-Textos.

García, B., Coronado, A., Montealegre, L. (2011, enero-abril). Formación y desarrollo de competencias matemáticas: una perspectiva teórica en la didáctica de las matemáticas. En Revista Educación y Pedagogía, 23(59), 159-175.

Gentili, P. (2015). América Latina, entre la desigualdad y la esperanza. Buenos Aires: Siglo XXI editores.

Ibáñez, J. (1979). Más allá de la sociología, el grupo de discusión. Técnica y crítica. España: 
Garzón C. (2020). El Saber Escolar Matemático En Colombia Y La Constitución De Subjetividades (Una Mirada Al Período Histórico 1995 - 2013). Revista Latinoamericana de Etnomatemática, 13(4), 120-

139. DOI: $10.22267 /$ relatem.20134.72

\section{Siglo XXI.}

Valero, P. (2002, enero-junio). Consideraciones sobre el contexto y la educación matemática para la democracia. Quadrante Vol 11 (1), 49-59

Valero, P. (2006, 24, 25 y 26 de octubre). ¿De carne y hueso? La vida social y política de la competencia matemática. [Mensaje en Foro Educativo Nacional]. Ministerio de Educación Nacional. Recuperado de http://www.colombiaaprende.edu.co/html/mediateca/1607/article-113423.html

Valero, P; y Skovsmose, O. (2012). Educación Matemática Crítica. Bogotá: Universidad de los Andes, Universidad de Aalborg.

Vithal, R., y Valero, P. (2012). La investigación en educación matemática en situaciones de conflicto social y político. En Valero, P., y Skovsmose, O. (Editores) Educación Matemática Crítica. Bogotá: Universidad de los Andes.

Trabal, P. (2011). Una Sociología de la enseñanza de las matemáticas, traducción del francés por Alejandro Rendón. Revista Educación y Pedagogía, 23(59), 227-240.

Zalamea, F. (2007). Fundamentos de las matemáticas. Bogotá: Universidad Nacional de Colombia

Zalamea, F. (2010). Razón de la frontera y fronteras de la razón. Universidad Nacional de Colombia.

Zalamea, F. (2014). Diagrama de las matemáticas hoy: el no lugar de Colombia en el panorama (Bogotá, Universidad Nacional de Colombia, Encuentro "250 años de Matemáticas - Homenaje a Mutis", Marzo 2012; repetida en Bogotá, Universidad Externado de Colombia, "Seminario de Historia y Educación Matemática", 30 de Septiembre 2014). https://www.etnomatematica.org/home/?p=4185

Zemelman, H. (2012). Pensar y poder, razonar y gramática del pensar histórico. Universidad de Ciencias y Artes de Chiapas: Siglo XXI Editores. 